\title{
Painless destructive thyroiditis in a patient with resistance to thyroid hormone: a case report
}

\author{
Tomoko Nagamine ${ }^{1 *}$ (D), Jaeduk Yoshimura Noh², Naoya Emoto' ${ }^{1}$, Takahito Kogai ${ }^{3}$, Akira Hishinuma ${ }^{3}$, \\ Fumitaka Okajima ${ }^{1}$ and Hitoshi Sugihara ${ }^{1}$
}

\begin{abstract}
Background: Resistance to thyroid hormone (RTH) usually features a syndrome of inappropriate secretion of thyroid-stimulating hormone (SITSH) without suppression of the typical high thyroid hormone levels. However, some patients with RTH show thyroid-stimulating hormone (TSH) suppression due to thyrotoxicosis. We report a case of painless thyroiditis in a patient with RTH that was misdiagnosed as Graves' disease because of TSHsuppressed thyrotoxicosis.

Case presentation: A sixteen-year-old boy consulted a local general physician for fatigue. He had a goiter, and biochemical analysis showed TSH $<0.1 \mu \mathrm{lU} / \mathrm{mL}$, free triiodothyronine (FT3) of $2.70 \mathrm{pg} / \mathrm{mL}$, and free thyroxine (FT4) of $3.6 \mathrm{ng} / \mathrm{dL}$. He was diagnosed with Graves' disease and was treated with $20 \mathrm{mg}$ thiamazole. One year later, he was referred to the department of endocrinology because of SITSH. He was finally diagnosed with RTH due to the finding of a heterozygous missense mutation (methionine 334 threonine) in the thyroid hormone receptor $\beta$ gene. Three years after cessation of thiamazole, his hyperthyroxinemia showed marked exacerbation with TSH suppression. We diagnosed him with painless destructive thyroiditis because of low technetium-99 m (Tc-99 m) uptake in the thyroid. Extreme hyperthyroxinemia was ameliorated, with a return to the usual SITSH levels, within 1 month without any treatment.

Conclusion: The present case demonstrates that diagnosing RTH is difficult when patients show hyperthyroxinemia with complete suppression of TSH to undetectable levels, and the data lead to misdiagnosis of RTH as Graves' disease. The initial diagnosis is important, and Tc-99 m scintigraphy is useful for the differential diagnosis of thyrotoxicosis accompanying RTH.
\end{abstract}

Keywords: Thyroid, Resistance to thyroid hormone, Painless thyroiditis, Scintigraphy

\section{Background}

RTH is an inherited syndrome of reduced tissue responsiveness to thyroid hormone [1-3]. RTH is characterized by elevated serum levels of FT4 or FT3 in the presence of high normal or slightly increased serum TSH concentrations. However, several reports have demonstrated that TSH is suppressed to very low or undetectable levels in patients with RTH coexisting with Graves' disease [4-8]. In these cases, a correct diagnosis is difficult

\footnotetext{
* Correspondence: nagamine@nms.ac.jp

'Department of Endocrinology, Diabetes and Metabolism, Graduate School of Medicine, Nippon Medical School, Tokyo, Japan

Full list of author information is available at the end of the article
}

if patients are not diagnosed with RTH beforehand. TSH levels may also be suppressed in patients with RTH in cases of thyrotoxic diseases other than Graves' disease. In this report, we present a patient with RTH who suffered from painless thyroiditis and whose TSH was completely suppressed to undetectable levels during transient hyperthyroxinemia.

\section{Case report}

A 16-year-old male with a goiter was referred to Nippon Medical School in May 2015. His medical history was unremarkable until 14 years of age (2013). His father and grandmother had hearing impairments. One year before

(c) The Author(s). 2019 Open Access This article is distributed under the terms of the Creative Commons Attribution 4.0 International License (http://creativecommons.org/licenses/by/4.0/), which permits unrestricted use, distribution, and 
the referral, the patient had complained of fatigue and a goiter and had consulted a family practitioner. A thyroid function test showed TSH $<0.1 \mu \mathrm{IU} / \mathrm{mL}$, FT3 of $2.70 \mathrm{pg} /$ $\mathrm{mL}$, and FT4 of $3.6 \mathrm{ng} / \mathrm{dL}$. The doctor misdiagnosed this thyrotoxicosis episode as Graves' disease without performing an ultrasound examination or confirming the presence of antithyroid antibodies and treated the patient with thiamazole $20 \mathrm{mg}$. Three months later, the patient showed hypothyroidism (TSH $265.7 \mu \mathrm{IU} / \mathrm{mL}$ and FT4 $0.4 \mathrm{ng} / \mathrm{dL}$ ) (Fig. 1), and the doctor reduced the thiamazole dose. However, SITSH (TSH 12.3-18.2 $\mu \mathrm{IU} / \mathrm{mL}$ and FT4 $2.0-2.1 \mathrm{ng} / \mathrm{dL}$ ) continued for 1 year, and the patient was referred to our hospital with a treatment plan of thiamazole at $5 \mathrm{mg}$ and $10 \mathrm{mg}$ doses alternating every other day. His thyroid function was indicated by TSH of $13.64 \mu \mathrm{IU} / \mathrm{mL}$, FT3 of $4.51 \mathrm{pg} / \mathrm{mL}$, and FT4 of $1.41 \mathrm{ng} / \mathrm{dL}$. We considered his SITSH to be a phenomenon of the transition from hypothyroidism to hyperthyroidism, and we thus decreased the thiamazole to $5 \mathrm{mg}$. His SITSH continued for the next 3 months, and magnetic resonance imaging (MRI) showed pituitary swelling. Because we could not exclude the possibility that the patient had TSHoma, we stopped thiamazole and admitted him to our ward for differential diagnosis of SITSH.

The patient's height was $158 \mathrm{~cm}$, and his body weight was $52.3 \mathrm{~kg}$. His temperature was 36.9 degrees, his heart rate was 83 beats per minute, and his blood pressure was $128 / 73 \mathrm{mmHg}$. In his usual SITSH state, thyroid ultrasonography showed a moderate goiter, slightly heterogeneous hypoechogenicity, and moderately rich blood flow (Fig. 2a). TSH receptor antibodies (TRAb), thyroid-stimulating autoantibodies (TSAb), and thyroidperoxidase antibodies (TPO-Ab) were all negative, and only anti-thyroglobulin antibodies ( $\mathrm{Tg}-\mathrm{Ab})$ were positive. The thyrotropin-releasing hormone test showed a normal TSH response, and the octreotide and bromocriptine test did not suppress the TSH levels. Sex hormone-binding globulin (SHBG) was within the normal range. These data suggested that his SITSH was unlikely to be due to TSHoma. Then, we examined whether the patient's family members had SITSH, and

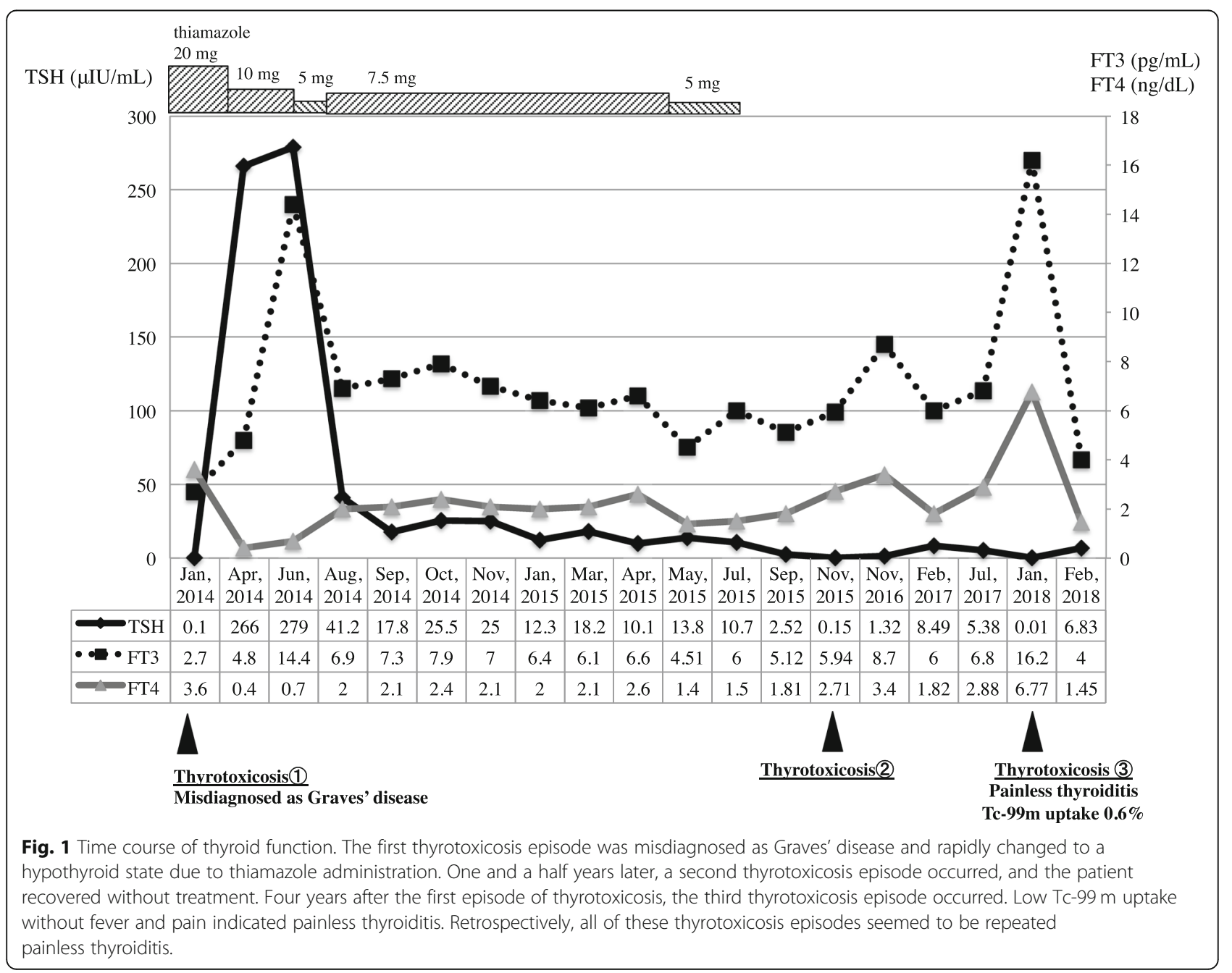


(a)
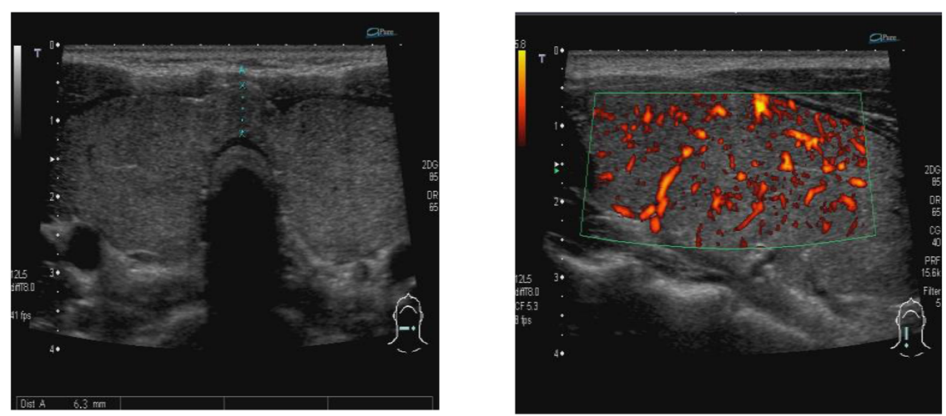

(b)
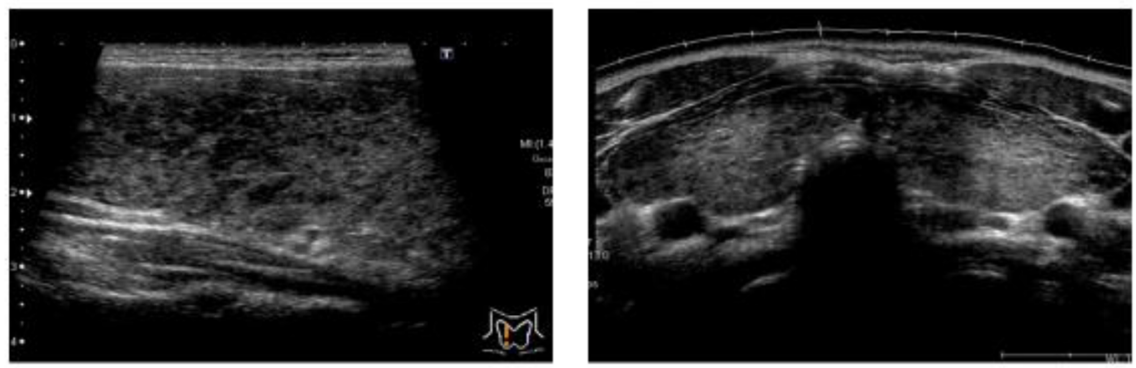

(c)
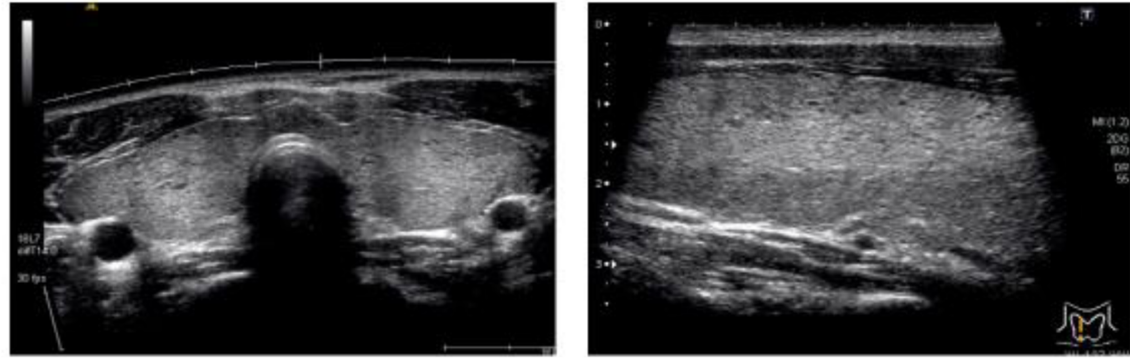

Fig. 2 Thyroid ultrasonography in each phase: (a) the SITSH state, while the patient was hospitalized in August 2015; (b) the third thyrotoxicosis episode in January 2018; and (c) recovery from painless thyroiditis in May 2019. (a) Moderate goiter, slightly heterogeneous hypoechogenicity, and moderately rich thyroid blood flow were detected. (b) There were focal hypoechoic areas without tenderness. (c) The hypoechoic area became invisible, and the echogenicity and homogeneity returned to the original condition

we identified SITSH in his father and his older brother. We found a genetic mutation of thyroid hormone receptor-beta (TR $\beta)$ exon 9, methionine 334 threonine, which was the same mutation reported by Mannavola et al. $[9,10]$, and we diagnosed the patient with RTH.

He developed a second case of thyrotoxicosis in November 2015, but it was mild and short. For the next two and a half years, his condition was stable as SITSH.

In January 2018, he complained of tachycardia and fatigue caused by a third episode of thyrotoxicosis. His TSH was suppressed, and TRAb remained negative. The technetium- $99 \mathrm{~m}$ (Tc-99 m) thyroid uptake was $0.6 \%$, which was low compared to his usual state (9.7\%). Therefore, we diagnosed this third episode of thyrotoxicosis as painless destructive thyroiditis. Thyroid ultrasonography during the third episode of thyrotoxicosis detected a moderate goiter, slightly heterogeneous hypoechogenicity, and moderately rich thyroid blood flow (Fig. 2b). The patient's thyroid function returned to his usual SITSH state without any treatment within 1 month. The hypoechoic area became invisible, and echogenicity and homogeneity returned to the original condition in January 2019, 16 months after the last episode of painless thyroiditis (Fig. 2c).

\section{Discussion}

We presented a case of repeated thyrotoxicosis with RTH. RTH usually features SITSH without suppression of the typical high thyroid hormone levels. However, some patients with RTH show TSH suppression during thyrotoxicosis episodes. Several reports have described patients with RTH complicated by thyrotoxic diseases, such as Graves' disease [4-8]. TSH levels can also be suppressed in patients with RTH in combination with thyrotoxic diseases other than Graves' disease. Taniyama et al. reported toxic multinodular goiter in patients with RTH [11] and episodes of repeated painless thyroiditis [12]. 
Painless thyroiditis usually occurs as a form of autoimmune thyroid disease, and the prevalence rate of thyroid autoantibodies is 2.36 times higher in RTH patients than in controls [13]. Furthermore, painless thyroiditis is more common in Japan than in the United States or Europe. We previously reported that the incidence of transient hyperthyroxinemia with suppressed Tc-99 $\mathrm{m}$ in hyperthyroid disease was $14.7 \%$ in a local area in Japan [14]. Other reports have shown that the incidence of painless thyroiditis was $10 \%$ in patients with hyperthyroid disease in Japan but was less than $1 \%$ in Denmark $[15,16]$. These results suggest that we must consider painless thyroiditis in RTH patients with thyrotoxicosis, especially in Japan. In our present case, a previous doctor misdiagnosed the first episode of thyrotoxicosis as Graves' disease, but painless thyroiditis could be considered as a differential diagnosis in terms of its frequency and the low FT3/FT4 ratio.

We think of these thyrotoxicosis episodes as repeated painless thyroiditis for four reasons. First, TRAb was negative at all times. Second, treatment with antithyroid drugs was nontherapeutic and caused severe hypothyroidism during the first episode of thyrotoxicosis. Third, the FT3/ FT4 ratio was low during all episodes of thyrotoxicosis. Finally, the Tc-99 m thyroid uptake was low, and the absence of fever or tenderness was not typical for subacute thyroiditis.

It is difficult to diagnose thyrotoxicosis with RTH. In general practice, thyroid scintigraphy is not available; therefore, the differential diagnosis of Graves' disease and other disorders depends on the existence of TRAb. Thyroid ultrasonography also provides useful data on thyroid size, tenderness, homogeneity, and blood flow. It was reported that thyroid blood flow is significantly higher in Graves' disease than in destructive thyroiditis. A thyroid blood flow of less than $4 \%$ suggests destructive thyroiditis [17]. In the present case, thyroid ultrasound was not performed during the first episode of thyrotoxicosis. Thyroid ultrasonography revealed a focal hypoechoic area during the third episode of thyrotoxicosis, and the hypoechoic region became invisible after recovery (Fig. 2b, c). There were no data on thyroid blood flow during thyrotoxicosis, but these changes in echogenicity suggested thyrotoxicosis after the destruction and regeneration of the thyroid.

When the patient was hospitalized in his usual SITSH state, his thyroid blood flow was relatively rich and was similar to that in mild Graves' hyperthyroidism. Because there are no sufficient thyroid ultrasonography data for $\mathrm{RTH}$, especially in the thyrotoxic state, thyroid ultrasounds seem to lack sufficient information for discriminating Graves' disease from other disorders. Moreover, TRAb can be positive in destructive thyroiditis. Therefore, only radioiodine or technetium uptake can provide a correct diagnosis of thyrotoxicosis with $\mathrm{RTH}$. Although it is difficult to determine the presence of RTH when patients show hyperthyroxinemia with complete suppression of TSH to undetectable levels, the initial diagnosis is important.

Compared with single episodes of thyroiditis, repeated painless thyroiditis is characterized by male gender, younger age, positive thyroid autoantibodies, and higher peak FT3 and FT4 levels [18]. Thyroiditis can occasionally develop in clusters in nursery school workers, and thus, an unidentified virus was hypothesized to be a cause of thyroiditis [19]. Because our patient always developed thyrotoxicosis in winter, some viral infection or seasonal allergy might have been a trigger. The association between RTH and repeated painless thyroiditis was unclear, but chronic thyroiditis, which often coexists with RTH, affected the patient's tendency toward painless thyroiditis.

\section{Conclusion}

We reported a case of repeated painless thyroiditis in a patient with RTH. RTH is known as a cause of SITSH and is rarely discovered in patients with thyrotoxicosis. It is difficult to treat thyrotoxicosis with RTH because these patients have FT3 and FT4 levels that are higher than normal. Therefore, the initial diagnosis is important during TSH suppression. Tc-99 $\mathrm{m}$ scintigraphy can detect the clinical condition of thyrotoxicosis regardless of whether the patient has concomitant RTH.

\begin{abstract}
Abbreviations
FT3: Free triiodothyronine; FT4: Free thyroxine; MRI: Magnetic resonance imaging; RTH: Resistance to thyroid hormone; SHBG: Sex hormone-binding globulin; SITSH: Syndrome of inappropriate secretion of thyroid-stimulating hormone; Tc-99 m: Technetium-99 m; Tg-Ab: Anti-thyroglobulin antibodies; TPO-Ab: Thyroid-peroxidase antibodies; TRAb: TSH receptor antibodies; TRB: Thyroid hormone receptor-beta; TSAb: Thyroid-stimulating autoantibodies; TSH: Thyroid-stimulating hormone
\end{abstract}

\section{Acknowledgments}

Not applicable.

Authors' contributions

All authors have read and approved the final version of the manuscript for publication.

Funding

Not applicable.

Availability of data and materials

Data sharing is not applicable for this article because no datasets were generated or analyzed during the current study.

Ethics approval and consent to participate

This case report was approved by the ethics committee of Ito Hospital.

Consent for publication

Consent was obtained from the patient and his parents.

Competing interests

The authors declare that they have no competing interests. 


\section{Author details}

'Department of Endocrinology, Diabetes and Metabolism, Graduate School of Medicine, Nippon Medical School, Tokyo, Japan. ${ }^{2}$ Department of Internal Medicine, Ito Hospital, Tokyo, Japan. ${ }^{3}$ Department of Infection Control and

Clinical Laboratory Medicine, Dokkyo Medical University, Tochigi, Japan.

Received: 4 April 2019 Accepted: 10 October 2019

Published online: 25 October 2019

\section{References}

1. Refetoff S, Weiss RE, Usala SJ. The syndromes of resistance to thyroid hormone. Endocr Rev. 1993;14(3):348-99.

2. Weiss RE, Refetoff S. Resistance to thyroid hormone. Rev Endocr Metab Disord. 2000;1(1-2):97-108.

3. Dumitrescu AM, Refetoff $\mathrm{S}$. The syndromes of reduced sensitivity to thyroid hormone. Biochim Biophys Acta. 2013;1830(7):3987-4003.

4. Sivakumar T, Chaidarun S. Resistance to thyroid hormone in a patient with coexisting Graves' disease. Thyroid. 2010;20(2):213-6.

5. Sato H. Clinical features of primary hyperthyroidism caused by Graves' disease admixed with resistance to thyroid hormone (P453T). Endocr $\mathrm{J}$. 2010;57(8):687-92.

6. Ogawa K, Yoshida M, Hayashi Y, Murata Y, Miyata M, Oiso Y. A rare case of resistance to thyroid hormone coexisting with Graves' disease. Endocrine. 2011:40(2):318-9.

7. Shiwa T, Oki K, Awaya T, Nakanishi S, Yamane K. Resistance to thyroid hormone accompanied by Graves' disease. Intern Med. 2011;50(18):1977-80.

8. Sun H, Xu S, Xie S, Cao W, Chen G, Di H, et al. Graves' disease coexisting with resistance to thyroid hormone: a rare case. Clin Case Rep. 2018;6(2):337-41.

9. Mannavola D, Vannucchi G, Fugazzola L, Cerutti N, Persani L, Beck-Peccoz P. Genetic analyses and evaluation of peripheral parameters of thyroid hormone action for the differential diagnosis of RTH. A novel heterozygous missense mutation (M334T) discovered. J Endocrinol Investig. 2002;25(2):Rc4-6.

10. Unluturk U, Sriphrapradang C, Erdogan MF, Emral R, Guldiken S, Refetoff S, et al. Management of differentiated thyroid cancer in the presence of resistance to thyroid hormone and TSH-secreting adenomas: a report of four cases and review of the literature. J Clin Endocrinol Metab. 2013;98(6): 2210-7.

11. Taniyama M, Ishikawa N, Momotani N, Ito K, Ban Y. Toxic multinodular goitre in a patient with generalized resistance to thyroid hormone who harbours the R429Q mutation in the thyroid hormone receptor beta gene. Clin Endocrinol. 2001;54(1):121-4.

12. Taniyama M, Otsuka F, Tozaki T, Ban Y. Thyroid profiles in a patient with resistance to thyroid hormone and episodes of thyrotoxicosis, including repeated painless thyroiditis. Thyroid. 2013;23(7):898-901.

13. Barkoff MS, Kocherginsky M, Anselmo J, Weiss RE, Refetoff S. Autoimmunity in patients with resistance to thyroid hormone. J Clin Endocrinol Metab. 2010;95(7):3189-93.

14. Kidokoro-Kunii Y, Emoto N, Cho K, Oikawa S. Analysis of the factors associated with Tc-99m pertechnetate uptake in thyrotoxicosis and graves' disease. J Nippon Med Sch. 2006;73(1):10-7.

15. Carle A, Pedersen IB, Knudsen N, Perrild H, Ovesen L, Rasmussen LB, et al. Epidemiology of subtypes of hyperthyroidism in Denmark: a populationbased study. Eur J Endocrinol. 2011;164(5):801-9.

16. Rasmussen NG, Hansen JM, Hegedus L. Frequency of thyroiditis and postpartum thyroiditis in a 10-year consecutive hyperthyroid Danish population. Thyroidology. 1989;1(3):143-7.

17. Ota H, Amino N, Morita S, Kobayashi K, Kubota S. Fukata S, et al. quantitative measurement of thyroid blood flow for differentiation of painless thyroiditis from Graves' disease. Clin Endocrinol. 2007;67:41-5.

18. Nishimaki M, Isozaki O, Yoshihara A, Okubo Y, Takano K. Clinical characteristics of frequently recurring painless thyroiditis: contributions of higher thyroid hormone levels, younger onset, male gender, presence of thyroid autoantibody and absence of goiter to repeated recurrence. Endocr J. 2009:56(3):391-7.

19. Ogura T, Hirakawa S, Suzuki S, Ota Z, Togawa T, Nogami I. Five patients with painless thyroiditis simultaneously developed in a nursery school. Endocrinol Jpn. 1988;35(2):225.

\section{Publisher's Note}

Springer Nature remains neutral with regard to jurisdictional claims in published maps and institutional affiliations.

Ready to submit your research? Choose BMC and benefit from:

- fast, convenient online submission

- thorough peer review by experienced researchers in your field

- rapid publication on acceptance

- support for research data, including large and complex data types

- gold Open Access which fosters wider collaboration and increased citations

- maximum visibility for your research: over $100 \mathrm{M}$ website views per year

At BMC, research is always in progress.

Learn more biomedcentral.com/submissions 